\section{Reconstituted epithelial corneal tissues for evaluation of drug delivery}

\author{
Susi Burgalassi, Erica Zucchetti, \\ Eleonora Terreni, Daniela Monti, \\ Silvia Tampucci, Patrizia Chetoni
}

Department of Pharmacy, University of Pisa, Pisa; Italian inter-university centre for the promotion of the 3Rs in teaching and research, Pisa, Italy

\begin{abstract}
One possible approach to reduce the use of animals in the evaluation of the ocular drug delivery is that to use cell cultures as model of tissues. In this study are compared two different reconstituted epithelial corneal tissues, a homemade artificial corneal epithelium (Reconstituted Rabbit Corneal Epithelium) and a commercial human corneal epithelial model (COR-100 EpiCornealTM, MatTek), for permeation experiments of three drugs with different physical chemical properties: timolol maleate, cyclosporin A, and a newly synthesized compound, MAGL 17.b. The collected data show that corneal epithelial models are not sufficient to simulate the complexity of the corneal barrier and the presence of a layer simulating the stroma may be necessary to approach the structure of native cornea.
\end{abstract}

\section{Introduction}

In the last few decades, new approaches have been used in research for the treatment of a large number of diseases, including the nanotechnologies, in order to prepare formulations/technologies for drug delivery. This great development of medical technologies has produced a significant increase of the number of animals used in research protocols, leading to an increasing demand to replace the in vivo methods with in vitro models. One possible approach to reduce the use of animals in the evaluation of drug delivery is that to use cell cultures as model of tissues. This approach is applied also in ocular field to study the biopharmaceutical behaviour of a lot of new drug delivery systems that have been developed to allow the drug to reach the different districts of the eye. Aim of this study was to compare different reconstituted epithelial corneal tissues for evaluation of ocular drug delivery: i) a homemade artificial corneal epithelium
(Reconstituted Rabbit Corneal Epithelium, $\mathrm{RRCE}^{1}$ and ii) a commercial human corneal epithelial model (COR-100 EpiCornealTM, MatTek). ${ }^{2}$

\section{Materials and Methods}

The rabbit corneal epithelial (RCE) cell line was obtained from the European Cell Culture Collection ( $\mathrm{N}^{\circ}$ 95081046, ECACC). To prepare RRCE, cells with passage numbers 12-16 were seeded onto specific polyester membranes (surface area 1.0 $\mathrm{cm}^{2}$ and pore size $0.4 \mu \mathrm{m}$; Snapwell ${ }^{\circledR}$, Costar) at a concentration of $8 \times 10^{4}$ cells $/ \mathrm{cm}^{2}$ and were grown at $37{ }^{\circ} \mathrm{C}$ in a humidified atmosphere containing $5 \% \mathrm{CO}_{2}$ with growth medium both in the apical and basolateral compartment, for 8 days until the cells had become confluent and were stratified. By removing the medium from the apical compartment, the cells were kept exposed to air for further 7 days changing daily the medium in the basolateral compartment up to obtain the reconstituted epithelium.

\section{Permeation experiments through RRCE, COR-100, and rabbit cornea}

For drug permeation experiments, the Snapwell ${ }^{\circledR}$ devices containing RRCE and the freshly excised rabbits' corneas were fitted into appropriated perfusion chambers, 3,4 consisting of two compartments (donor and receiving) separated by the tissue while COR-100 was tested into its native support (Transwell ${ }^{\circledR}$ plate).

The drugs employed in the study were timolol maleate (TM), cyclosporin A (CyA), and a newly synthesized compound, $M A G L$ 17.b. The physical chemical properties of the different molecules are listed in Table 1. The donor phases of the permeation experiments were aqueous solutions containing drug plus i) permeation enhancers (surfactants) and ii) nanostructured systems as nanomicelles.

\section{Trans-epithelial electrical resistance}

The trans-epithelial electrical resistance (TEER) was measured during RCE cell growth using an EVOM epithelial voltohmmeter to evaluate the integrity of the cell layers and their tightness. TEER was also

Table 1. Physical chemical properties of the different molecules.

\begin{tabular}{lccc} 
Drug & MW $(\mathrm{Da})$ & $\log \mathrm{P}$ o/w & Vehicle \\
TM & 432 & 1.4 & surfactants \\
CyA & 1202 & $2.8-3.6$ & nanomicelles \\
\hline MAGL 17.b & 343 & 3.4 & surfactants \\
\hline
\end{tabular}

Correspondence: Susi Burgalassi, Department of Pharmacy, University of Pisa, via Bonanno 6, 56126 Pisa, Italy.

E-mail: susi.burgalassi@unipi.it

Key words: Corneal permeation; reconstituted tissues; drug partition coefficient.

Conference presentation: this paper was presented at the Second Centro 3R Annual Meeting - 3Rs in Italian Universities, 2019, June 20-21, University of Genoa, Italy.

Received for publication: 28 October 2019. Accepted for publication: 6 November 2019.

This work is licensed under a Creative Commons Attribution NonCommercial 4.0 License (CC BY-NC 4.0).

(C) Copyright: the Author(s), 2019

Licensee PAGEPress, Italy

Biomedical Science and Engineering 2019; 3(s2): doi:10.4081/bse.2019.82

always measured before and at end of each permeation experiment to verify the integrity of the membranes.

\section{Results}

The results of permeation experiments were reported as apparent permeability coefficient $\left(\mathrm{P}_{\mathrm{app}}\right)$ defined by the equation:

$P_{\text {app }}=(\mathrm{dQ} / \mathrm{dt}) /\left(\mathrm{AC}_{0}\right)$

where $\mathrm{A}$ and $\mathrm{C}_{0}$ are the exposed membrane surface area and the initial drug concentration, respectively. $\mathrm{P}_{\text {app }}$ was calculated from slopes of linear plots of amount of drug in the receiving chamber $(\mathrm{Q}) v s$ time $(\mathrm{t})$ at the steady-state.

The close similarity of $\mathrm{P}_{\text {app }}$ values obtained with RRCE and isolated corneas in permeation experiments with TM (the most hydrophilic among the drugs tested) points to the analogy of barriers to drug permeation present in the two substrates. When the lipophilic properties of the molecule increase the stroma becomes the barrier limiting the permeation through the cornea and the epithelial models become inadequate to simulate the ocular barrier and a 
corrective value should be introduced. From the toxicity point of view, in every case a decrease of the TEER values measured before and after trans-epithelial permeation experiments was evident, regardless of the type of product used, indicating some degree of suffering of the reconstituted epithelial corneal tissues caused by the test conditions.

\section{Conclusions}

The collected data show that corneal epithelial models are not sufficient to simu- late the complexity of the corneal barrier and the presence of a layer simulating the stroma may be necessary to approach the structure of native cornea.

Moreover, the epithelial models were more sensitive than native cornea to the permeation experiment conditions.

\section{References}

1. Burgalassi S, Monti D, Brignoccoli A, et al. Development of cultured rabbit corneal epithelium for drug permeation studies: a comparison with excised rab- bit cornea. J Ocular Pharmacol Ther 2004;20:518-32.

2. Kaluzhny Y, Kinuthia MW, Truong T, et al. New human organotypic corneal tissue model for ophthalmic drug delivery studies. Invest Ophthalmol Vis Sci 2018;59:2880-98.

3. Grass GM, Sweetana SA. In vitro measurement of gastrointestinal tissue permeability using a new diffusion cell. Pharm Res 1988;5:372-6.

4. Camber O. An in vitro model for determination of drug permeability through the cornea. Acta Pharm Suec 1985;22:33542. 